\title{
Fuzzy Dot Satisfactory Bck- Filters
}

\author{
S. A. Najati \\ Department of Mathematics and Statistics, Taif University, Taif, KSA
}

\begin{abstract}
The concept of fuzzy dot subalgebra of $B C K / B C I$-algebras was introduced by Jun and Hong paper [8]. In 2003 Jun [7] was introduced the concept of satisfactory $B C K$-filters. The concept of fuzzy satisfactory $B C K$-filters was introduced by Najati [13]. In this paper, we define the notion of fuzzy dot satisfactory $B C K$-filters and investigate some of its properties.
\end{abstract}

Keywords: BCK-algebras, filter, dot filter, satisfactory filter, fuzzy filter, fuzzy dot filter, fuzzy satisfactory filter, fuzzy dot satisfactory filter.

\section{INTRODUCTION}

The notion of BCK-algebra was introduced by Imai and Iseki in 1966 [5]. In the same year, Iseki [6] introduced the notion of BCI-algebra which is a generalization of a BCK-algebra. After the introduction of the concept of fuzzy sets by Zadeh [17], several researchers were conducted on the generalization of the notion of fuzzy sets. Jun and Hong in [8] introduced the notion of a fuzzy dot subalgebra of a BCK/BCI-algebras as a generalization of a fuzzy subalgebra and state a condition for a fuzzy dot subalgebra and prove several basic properties which are related to fuzzy dot subalgebra. In [12] Najati introduced the notion of fuzzy dot filters in BCK-algebras and investigate some of its properties. In [13] Najati introduced the notion of a fuzzy satisfactory filter in BCKalgebra and prove several basic properties which are related to fuzzy satisfactory filter. In this paper, we introduce the notion of fuzzy dot satisfactory BCK-filter and prove some their fundamental properties, and then we state a condition for a fuzzy subset to be a fuzzy dot satisfactory filter.

\section{Preliminaries}

We review some basic definitions and properties that will be useful in our results. A $B C K$-algebra $X$ is defined to be an algebra $(X, *, 0)$ of type $(2,0)$ satisfying the following conditions.

$B C K-1((x y)(x z))(z y)=0$,
$B C K-2(x(x y)) y=0$,

$B C K-3 x x=0$,

$B C K-40 x=0$,

$B C K-5 x y=0$ and $y x=0 \Rightarrow x=y$,

for all $x, y, z \in X$, where $x y=x * y$, and $x y=0$ if and only if $x \leq y$.

In a $B C K$-algebra $X$, the following properties hold for all $x, y, z \in X$ :

$\mathrm{P}-1 x 0=x$,

$\mathrm{P}-2(x y) z=(x z) y$,

P-3 $x \leq y$ implies that $x z \leq y z$ and $z y \leq z x$,

$\mathrm{P}-4(x z)(y z) \leq x y$,

P-5 $x \leq y, y \leq z \Rightarrow x \leq z$,

P-6 $x(x(x y))=x y$,

$\mathrm{P}-7 x y \leq x$.

A $B C K$-algebra $X$ satisfying the identity $x(x y)=$ $y(y x)$, for all $x, y \in X$, is said to be commutative. If there is a special element 1 in a $B C K$-algebra $X$ satisfying $x \leq$ 1 , for all $x \in X$, then 1 is called a unit of $X$. A $B C K$ algebra $X$ with unit is said to be bounded. In what follows let $X$ denote a bounded $B C K$-algebra unless otherwise specified, and we will use the notation $x^{*}$ instead $1 x$ for all $x \in X$. In a bounded $B C K$-algebra $X$ we have:

$\mathrm{P}-8 \quad 1^{*}=0$ and $0^{*}=1$.

P-9 $y \leq x$ implies that $x^{*} \leq y^{*}$. 
$\mathrm{P}-10 x^{*} y^{*} \leq y x$.

If $X$ is a commutative bounded $B C K$-algebra, then the equalities $\left(x^{*}\right)^{*}=x, x^{*} y^{*}=y x$ hold, for all $x, y \in X$.

We review some fuzzy concepts. A fuzzy subset of a nonempty set $X$ is a function $\mu: X \rightarrow[0,1]$. We shall use the notation $X_{\mu}$ for $\{x \in X \mid \mu(x)=\mu(1)\}$. The set $\mu_{t}=$ $\{x \in X \mid \mu(x) \geq t\}$, where $t \in[0,1]$, is called the t-level subset of $\mu$.

A nonempty subset of $X$ Then $F$ is called a filter of $X$, if it satisfies the conditions.

$\mathrm{F}-11 \in X$,

F-2 $\left(x^{*}\right)^{*} y^{*} \in F, y \in F \Rightarrow x \in F$ for all $x, y \in X$.

A nonempty subset $F$ of $X$ is called a satisfactory filter of $X$, if it satisfies (F-1) and

F-3 $\left(x\left(y(y z)^{*}\right)^{*}\right)^{*} \in F, x \in F \Rightarrow(y z)^{*} \in F$, for all $x, y, z \in X$.

A $B C K$-algebra $X$ satisfying $(x z)(y z)=(x y) z$, for all $x, y, z \in X$, is said to be positive implicative. A $B C K$ algebra $X$ is positive implicative if and only if it satisfies $x y=(x y) y$, for all $x, y \in X$.

A fuzzy subset $\mathcal{F}$ in $X$ is said to be a fuzzy filter of $X$, if it satisfies:

FF-1 $\mathcal{F}(1) \geq \mathcal{F}(x)$,

FF-2 $\mathcal{F}(x) \geq \min \left\{\mathcal{F}\left(\left(x^{*} y^{*}\right)^{*}\right), \mathcal{F}(y)\right\}$, for all $x, y \in X$.

Note that every fuzzy filter is order preserving (see [9, Proposition 3.5]).

Definition 2.1. [12] A fuzzy subset $\mathcal{F}$ in $X$ is said to be a fuzzy dot filter of $X$, if it satisfies FF-1 and, FF-3 $\mathcal{F}(x) \geq \mathcal{F}\left(\left(x^{*} y^{*}\right)^{*}\right) \mathcal{F}(y)$, for all $x, y \in X$.

Theorem 2.2. [12, Theorem 3.7] Let $\mathcal{F}$ be a fuzzy subset of $X$ and $\mathcal{F}(1)=1$. Then

$$
\begin{gathered}
\mathcal{F}(\boldsymbol{x} \boldsymbol{z})^{*} \geq \mathcal{F}(\boldsymbol{y}) \text { implies } \mathcal{F}(\boldsymbol{z}) \geq \mathcal{F}(\boldsymbol{y}), \\
\boldsymbol{x} \boldsymbol{y} \leq \boldsymbol{z} \text { implies } \mathcal{F}(\boldsymbol{y}) \geq \mathcal{F}(\boldsymbol{x}) \mathcal{F}\left(\boldsymbol{z}^{*}\right)
\end{gathered}
$$

Theorem 2.3. Let $X$ be commutative and let $\mathcal{F}$ be a fuzzy dot filter of $X$. Then for all $x, y \in X$

$$
\left.\mathcal{F}(x) \geq \mathcal{F}\left((x y)^{*} x\right)^{*}\right)
$$

Proof. Let $\mathcal{F}$ be a fuzzy dot filter of $X$. Then for all $x, y, z \in X$, we get:

$$
\begin{aligned}
\left((x y)^{*} x\right)^{*}(y x)^{*} & =(y x)\left((x y)^{*} x\right) \\
& \leq y(x y)^{*} \\
& =(x y) y^{*} \\
& =(x y)(1 y) \\
& \leq x 1
\end{aligned}
$$

$$
=0
$$

Then

$$
\mathcal{F}\left((x y)^{*} x\right)^{*} \leq \mathcal{F}(y x)^{*}
$$

Then by Theorem 2.2 we get:

$$
\left.\mathcal{F}(x) \geq \mathcal{F}\left((x y)^{*} x\right)^{*}\right)
$$

Definition 2.4. [13] A fuzzy subset $\mathcal{F}$ in $X$ is said to be a fuzzy satisfactory filter of $X$, if it satisfies FF-1, and FF-4 $\mathcal{F}\left((y z)^{*}\right) \geq \min \left\{\mathcal{F}\left(\left(x\left(y(y z)^{*}\right)^{*}\right)^{*}\right), \mathcal{F}(x)\right\}$, for all $x, y, z \in X$.

\section{FUZZY DOT SATISFACTORY FILTERS}

Definition 3.1. A fuzzy subset $\mathcal{F}$ in $X$ is said to be a fuzzy dot satisfactory filter of $X$, if it satisfies FF-1, and FF-5 $\mathcal{F}\left((y z)^{*}\right) \geq \mathcal{F}\left(\left(x\left(y(y z)^{*}\right)^{*}\right)^{*}\right) \mathcal{F}(x)$, for all $x, y, z \in X$.

Example 3.2. Let $X=\{0, a, b, 1\}$ be a bounded $B C K$ algebra with $*$ defined by

\begin{tabular}{|c|c|c|c|c|}
\hline$*$ & 0 & $a$ & $b$ & 1 \\
\hline 0 & 0 & 0 & 0 & 0 \\
\hline$a$ & $a$ & 0 & $a$ & 0 \\
\hline$b$ & $b$ & $b$ & 0 & 0 \\
\hline 1 & 1 & $b$ & $a$ & 0 \\
\hline
\end{tabular}

Define $\mathcal{F}$ of $X$ by $\mathcal{F}(1)=0.4$ and $\mathcal{F}(0)=\mathcal{F}(a)=$ $\mathcal{F}(b)=0.3$. Routine calculations give that $\mathcal{F}$ is not a fuzzy filter of $X$. Also, a fuzzy subset $v$ of $X$ defined by $v(0)=0.3, v(1)=v(b)=0.5$, and $v(a)=0.6$. Routine calculations give that $v$ is not a fuzzy filter of $X$.

Remark 3.3. Every fuzzy satisfactory filter of $X$ is a fuzzy dot satisfactory filter of $X$, since:

$$
\begin{aligned}
\mathcal{F}\left((x z)^{*}\right) & \geq \min \left\{\mathcal{F}\left(\left(x\left(y(y z)^{*}\right)^{*}\right)^{*}\right), \mathcal{F}(x)\right\} \\
& \geq \mathcal{F}_{F}\left(\left(x\left(y(y z)^{*}\right)^{*}\right)^{*}\right) \mathcal{F}(x)
\end{aligned}
$$

But the converse may not be true as seen in above example, since $v$ is a fuzzy dot satisfactory filter of $X$, but it is not a fuzzy satisfactory filter because:

$$
\begin{aligned}
v(10)^{*} & =0.3 \\
& <\min \left\{v\left(\left(b\left(1(10)^{*}\right)^{*}\right)^{*}\right), v(b)\right\} \\
& =\min \{v(a), v(b)\}=0.5
\end{aligned}
$$

Remark 3.4. If $F$ be a nonempty satisfactory filter of $X$, and the fuzzy set $\mathcal{F}_{F}$ of $X$, which defined by $\mathcal{F}_{F}(x)=s$ if $x \in F$ and $\mathcal{F}_{F}(x)=t$ if $x \notin F$, for all $s, t \in[0,1]$ with $s>t$, then by [13, Theorem 4.4] $\mathcal{F}_{F}$ is a fuzzy satisfactory filter of $X$. So, by above remark $\mathcal{F}_{F}$ is a fuzzy dot satisfactory filter of $X$. In the following theorem we refer this remark with another proof. 
Theorem 3.5. Let $F$ be a nonempty satisfactory filter of $X$ and let the fuzzy set $\mathcal{F}_{\mathrm{F}}$ of $X$, which defined by $\mathcal{F}_{F}(x)=s$ if $x \in F$ and $\mathcal{F}_{F}(x)=t$ if $x \notin F$, for all $s, t \in$ $[0,1]$ with $s>t$, then it is a fuzzy dot satisfactory filter of $X$.

Proof. Let $F$ be a nonempty satisfactory filter of $X$, since $1 \in F$, we have $\mathcal{F}(1)=s \geq \mathcal{F}(x)$ for all $x \in X$. Now let $x, y, z \in X$. If $\left(x\left(y(y z)^{*}\right)^{*}\right)^{*} \in F$ and $x \in F$, then $(y z)^{*} \in F$, then

$$
\mathcal{F}_{F}(y z)^{*}=s \geq \mathcal{F}_{F}\left(\left(x\left(y(y z)^{*}\right)^{*}\right)^{*}\right) \mathcal{F}_{F}(x)
$$

If $\left(x\left(y(y z)^{*}\right)^{*}\right)^{*} \notin F$ or $x \notin F$, then

$$
\mathcal{F}_{F}\left(\left(x\left(y(y z)^{*}\right)^{*}\right)^{*}\right) \mathcal{F}_{F}(x)=s t \leq \mathcal{F}_{F}(y z)^{*}
$$

It follows that $\mathcal{F}_{F}$ is a fuzzy dot satisfactory filter of $X$.

Theorem 3.6. Let $\mathcal{F}$ be a fuzzy dot satisfactory filter of $X$, then $X_{\mathcal{F}}=\{x \in X \mid \mathcal{F}(x)=1\}$ is either empty or satisfactory filter of $X$.

Proof. Suppose that $\mathcal{F}$ is a fuzzy dot satisfactory filter of $X$, clearly $1 \in X_{\mathcal{F}}$. Now let $X_{\mathcal{F}} \neq \emptyset$. Let $x, y, z \in X$, and let $\left(x\left(y(y z)^{*}\right)^{*}\right)^{*} \in X_{\mathcal{F}}$ and $x \in X_{\mathcal{F}}$, then:

$$
\mathcal{F}(y z)^{*} \geq \mathcal{F}\left(\left(x\left(y(y z)^{*}\right)^{*}\right)^{*}\right) \mathcal{F}(x)=1
$$

So $(y z)^{*} \in X_{\mathcal{F}}$, then $X_{\mathcal{F}}$ is a satisfactory filter of $X$.

Proposition 3.7. Let $\mathcal{F}$ be a fuzzy dot satisfactory filter of $X$. Then for all $x \in X, \mathcal{F}(0) \geq \mathcal{F}\left(x^{*}\right) \mathcal{F}(x)$.

Proof. Let $x \in X$, then:

$\mathcal{F}(0)=\mathcal{F}(10)^{*} \geq \mathcal{F}\left(x\left(1(10)^{*}\right)^{*} \mathcal{F}(x)=\mathcal{F}\left(x^{*}\right) \mathcal{F}(x)\right.$.

Lemma 3.8. [7, Theorem 3.13] Let $X$ be commutative and $G^{*}=\left\{x^{*} \mid x \in G\right\}$ for every nonempty subset $G$ of $X$ . Then $G^{*}$ is a satisfactory filter of $X$ if and only if $G$ is a positive implicative ideal of $X$.

Theorem 3.9. Assume that $X$ is commutative. Let $G$ be a nonempty subset of $X$ and $\mathcal{F}_{G^{*}}$ a fuzzy set in $X$ defined by:

$$
\mathcal{F}_{G^{*}}=\left\{\begin{array}{cc}
s & \text { if } x \in G^{*}=\left\{x^{*} \mid x \in G\right\} \\
t & \text { otherwise, }
\end{array}\right.
$$

for all $x \in X$ and $0 \leq t<s \leq 1$. If $G$ is a positive implicative ideal of $X$, then $\mathcal{F}_{G^{*}}$ is a fuzzy dot satisfactory filter of $X$.

Proof. Assume that $G$ is a positive implicative ideal of $X$ , then by Lemma 3.8. $G^{*}$

is a satisfactory filter of $X$, So $\mathcal{F}_{G^{*}}$ is a fuzzy dot satisfactory filter of $X$ by Theorem 3.5.

Theorem 3.10. Let $\mathcal{F}$ be a fuzzy set of $X$. If $\mathcal{F}_{\mathrm{t}}$ is a satisfactory filter of $X$ for all $t \in[0,1]$ where $\mathcal{F}_{\mathrm{t}} \neq \varnothing$, then $\mathcal{F}$ is a fuzzy dot satisfactory filter of $X$.

Proof. Assume that $\mathcal{F}_{\mathrm{t}}$ is a satisfactory filter of $X$ for all $t \in[0,1]$, and $\mathcal{F}_{\mathrm{t}} \neq \emptyset$, we claim that (FF-1) and (FF-4) are true. If (FF-1) is not true, then there is $x_{0} \in X$ be such that, $\mathcal{F}(1)<\mathcal{F}\left(x_{0}\right)$. Put $t_{0}=\frac{1}{2}\left\{\mathcal{F}(1)+\mathcal{F}\left(x_{0}\right)\right\}$. Then $t_{0} \in$
$[0,1]$ and $\mathcal{F}(1)<t_{0}<\mathcal{F}\left(x_{0}\right)$, which implies that $x_{0} \in$ $\mathcal{F}_{t_{0}}$ and $\mathcal{F}_{t_{0}} \neq \emptyset$. So $\mathcal{F}_{t_{0}}$ is a satisfactory filter of $X$ by assumption. It follows from $1 \in \mathcal{F}_{t_{0}}$ that $\mathcal{F}(1) \geq t_{0}$ which is a contradiction. Therefore (FF-1) holds. Suppose that (FF-4) is false. Then there are $x_{0}, y_{0}, z_{0} \in X$ such that:

Taking

$$
\mathcal{F}\left(\left(y_{0} z_{0}\right)^{*}\right)<\mathcal{F}\left(\left(x_{0}\left(y_{0}\left(y_{0} z_{0}\right)^{*}\right)^{*}\right)^{*}\right) \mathcal{F}\left(x_{0}\right)
$$

$s_{0}=\frac{1}{2}\left\{\mathcal{F}\left(\left(y_{0} z_{0}\right)^{*}\right)+\mathcal{F}\left(\left(x_{0}\left(y_{0}\left(y_{0} z_{0}\right)^{*}\right)^{*}\right)^{*}\right) \mathcal{F}\left(x_{0}\right)\right\}$,

we get $s_{0} \in[0,1]$ and

$$
\mathcal{F}\left(\left(y_{0} z_{0}\right)^{*}\right)<s_{0}<\mathcal{F}\left(\left(x_{0}\left(y_{0}\left(y_{0} z_{0}\right)^{*}\right)^{*}\right)^{*}\right) \mathcal{F}\left(x_{0}\right)
$$

It follows from the right-hand side of the above inequality that:

$$
\left(x_{0}\left(y_{0}\left(y_{0} z_{0}\right)^{*}\right)^{*}\right)^{*} \in \mathcal{F}_{S_{0}} \text { and } x_{0} \in \mathcal{F}_{S_{0}}
$$

Since $\mathcal{F}_{S_{0}}$ is a satisfactory filter of $X$ it follows form $\left(y_{0} z_{0}\right)^{*} \in \mathcal{F}_{s_{0}}$, that $\mathcal{F}\left(\left(y_{0} z_{0}\right)^{*}\right) \geq s_{0}$ so, which is impossible. Hence (FF-4) is also valid. Consequently, $\mathcal{F}$ is a fuzzy dot satisfactory filter of $X$.

Theorem 3.11. [12, Theorem 3.8] Let $X$ be a commutative and $\mathcal{F}$ and a fuzzy subset of $X$. Then $\mathcal{F}$ fuzzy dot filter of $X$ if and only if it satisfies for all $x, y \in X, \mathcal{F}(x) \geq \mathcal{F}(y x)^{*} \mathcal{F}(y)$.

Theorem 3.12. If $X$ is commutative, then every fuzzy dot satisfactory filter of $X$ is a fuzzy dot filter of $X$

Proof. Let $\mathcal{F}$ be a fuzzy dot satisfactory filter of $X$ and $x, y \in X$. Since $x^{* *}=x$, we get $(x y)^{*}=\left(x\left(y^{* *}\right)^{* *}\right)^{*}$. it follows from FF-5 that:

$$
\begin{aligned}
\mathcal{F}(y) & =\mathcal{F}\left(y^{* *}\right) \\
& \geq \mathcal{F}\left(x\left(1(1 y)^{*}\right)^{*}\right)^{*} \mathcal{F}(x) \\
& =\mathcal{F}(x y)^{*} \mathcal{F}(x)
\end{aligned}
$$

So, from Theorem 3.10. that $\mathcal{F}$ is a fuzzy dot filter of $X$.

The converse of above theorem may not be true as seen in the following example.

Example 3.13. Let $X=\{0, a, b, 1\}$ be a bounded $B C K$ algebra with $*$ defined by:

\begin{tabular}{|c|c|c|c|c|}
\hline$*$ & 0 & $a$ & $b$ & 1 \\
\hline 0 & 0 & 0 & 0 & 0 \\
\hline$a$ & $a$ & 0 & 0 & 0 \\
\hline$b$ & $b$ & $a$ & 0 & 0 \\
\hline 1 & 1 & $b$ & $a$ & 0 \\
\hline
\end{tabular}

Define $\mathcal{F}$ of $X$ by $\mathcal{F}(1)=1$ and $\mathcal{F}(x)=t, t \in[0,1]$ for all $x \neq 1$. Routine calculations give that $\mathcal{F}$ is a fuzzy dot filter of $X$, but it is not fuzzy dot satisfactory filter of $X$, because.

$$
\mathcal{F}(b a)^{*}=t \geq 1=\mathcal{F}\left(\left(1\left(b(b a)^{*}\right)^{*}\right)^{*}\right) \mathcal{F}(1)
$$

Theorem 3.14. [12, Proposition 3.4] Every fuzzy dot filter $\mathcal{F}$ of $X$ with $\mathcal{F}(1)=1$ is order preserving. 
Remark 3.15. By Theorem 3.12 and Theorem 3.14 if $X$ is commutative, and $\mathcal{F}$ is a fuzzy dot satisfactory filter of $X$ with $\mathcal{F}(1)=1$, then it is a fuzzy dot filter, so it is preserving.

We give conditions for a fuzzy dot filter to be a fuzzy dot satisfactory filter of $X$.

Theorem 3.16. Let $X$ be a commutative and positive implicative. Then every fuzzy dot filter of $X$ is a fuzzy dot satisfactory filter of $X$.

Proof. Let $\mathcal{F}$ be a fuzzy dot filter of $X$. Since

$(y z)^{*}=\left(z^{*} y^{*}\right)^{*}=\left(\left(z^{*} y^{*}\right) y^{*}\right)^{*}=\left((y z) y^{*}\right)^{*}=$

$\left(y(y z)^{*}\right)^{*}$ for all $y, z \in X$, it follows from Theorem 3.10.

$\mathcal{F}\left((y z)^{*}\right)=\mathcal{F}\left(\left(y(y z)^{*}\right)^{*}\right) \geq \mathcal{F}\left(\left(x\left(y(y z)^{*}\right)^{*}\right)^{*}\right) \mathcal{F}(x)$

So that $\mathcal{F}$ is a fuzzy dot satisfactory filter of $X$.

Theorem 3.17. Let $X$ be a commutative and let $\mathcal{F}$ be a fuzzy dot satisfactory filter of $X$ where $\mathcal{F}(1)=1$. Then for all $x, y \in X$

$$
\mathcal{F}\left((x y)^{*}\right) \geq \mathcal{F}\left(\left(x(x y)^{*}\right)^{*}\right)
$$

Proof. Let $\mathcal{F}$ be a fuzzy dot satisfactory filter of $X$, then

$$
\begin{aligned}
\mathcal{F}\left((x y)^{*}\right) & \geq \mathcal{F}\left(\left(1\left(x(x y)^{*}\right)^{*}\right)^{*}\right) \mathcal{F}(1) \\
& =\mathcal{F}\left(\left(\left(\left(x(x y)^{*}\right)^{*}\right)^{*}\right)^{*}\right) \\
& =\mathcal{F}\left(\left(x(x y)^{*}\right)^{*}\right)
\end{aligned}
$$

for all $x, y \in X$. Therefore (1) holds.

Theorem 3.18. Every fuzzy dot filter of $X$ satisfying (1) is a fuzzy dot satisfactory filter of $X$.

Proof. Let $\mathcal{F}$ be a fuzzy dot filter of $X$ satisfying (1), then by Theorem 3.11.

$\mathcal{F}\left((y z)^{*}\right)=\mathcal{F}\left(\left(y(y z)^{*}\right)^{*}\right) \geq \mathcal{F}\left(\left(x\left(y(y z)^{*}\right)^{*}\right)^{*}\right) \mathcal{F}(x)$

for all $x, y, z \in X$. Therefore, $\mathcal{F}$ is a fuzzy dot satisfactory filter of $X$.

Theorem 3.19. Let $X$ be a commutative and let $\mathcal{F}$ be a fuzzy dot filter of $X$. Then for all $x, y \in X$

$$
\left.\mathcal{F}(x) \geq \mathcal{F}\left((x y)^{*} x\right)^{*}\right)
$$

Proof. Let $\mathcal{F}$ be a fuzzy dot filter of $X$, then:

$$
\begin{aligned}
\left((x y)^{*} x\right)^{*} & =(y x)\left((x y)^{*} x\right) \\
& \leq y(x y)^{*} \\
& =(x y) y^{*} \\
& =(x y)(1 y) \\
& \leq x 1 \\
& =0
\end{aligned}
$$

for all $x, y \in X$, then $\mathcal{F}\left((x y)^{*} x\right)^{*} \leq \mathcal{F}(y x)^{*}$, then by Theorem 2.2 (i) we get:

$$
\left.\mathcal{F}(x) \geq \mathcal{F}\left((x y)^{*} x\right)^{*}\right)
$$

Theorem 3.20. Let $X$ be a commutative and let $\mathcal{F}$ be a fuzzy dot filter of $X$ where $\mathcal{F}(1)=1$. Then the following are equivalent for all $x, y, z \in X$ i. $\mathcal{F}$ is a fuzzy dot satisfactory filter of $X$,

ii. $\left.\quad \mathcal{F}(y) \geq \mathcal{F}\left(\left(x(y z)^{*} y\right)\right)^{*}\right) \mathcal{F}(x)$.

Proof. $(i) \Rightarrow$ (ii) Let $\mathcal{F}$ be a fuzzy dot satisfactory filter of $X$, by Theorem 3.11 and Theorem 3.19 we have $\left.\mathcal{F}(y) \geq \mathcal{F}\left((y z)^{*} y\right)^{*}\right) \geq \mathcal{F}\left(x\left((y z)^{*} y\right)^{*}\right) \mathcal{F}(x)$

Thus (ii) is valid.

(ii) $\Rightarrow$ (i) Assume that a fuzzy dot filter $\mathcal{F}$ of $X$ satisfies the condition (ii), for all $x, y \in X$, it follows from (ii) that:

Thus

$$
\mathcal{F}(x) \geq \mathcal{F}\left(\left(\left((x y)^{*} x\right)^{*}\right)^{* *}\right) \mathcal{F}(1) \geq \mathcal{F}\left(\left((x y)^{*} x\right)^{*}\right)
$$

$$
\mathcal{F}(x) \geq \mathcal{F}\left(\left((x y)^{*} x\right)^{*}\right)
$$

for all $x, y \in X$. Taking $x=(x y)^{*}$ in $\left(1^{*}\right)$ implies that

$$
\mathcal{F}(x y)^{*} \geq \mathcal{F}\left(\left((x y)^{*} y\right)^{*}(x y)^{*}\right)^{*}
$$

On the other hand, note that:

$$
\begin{aligned}
\left(\left((x y)^{*} y\right)^{*}(x y)^{*}\right)^{*} & =\left((x y)\left((x y)^{*} y\right)\right)^{*} \\
& =\left(\left(y^{*} x^{*}\right)\left(y^{*}(x y)\right)\right)^{*} \\
& =\left(\left(y^{*}\left(y^{*}(x y)\right)\right) x^{*}\right)^{*} \\
& =\left(\left((x y)\left((x y) y^{*}\right)\right) x^{*}\right)^{*} \\
& =\left(\left(\left(y^{*} x^{*}\right)\left(\left(y^{*} x^{*}\right) y^{*}\right)\right) x^{*}\right)^{*} \\
& =\left(\left(\left(y^{*} x^{*}\right) x^{*}\right)\left(\left(y^{*} x^{*}\right) y^{*}\right)\right)^{*} \\
& =\left(\left(\left(y^{*} x^{*}\right) x^{*}\right)\left(\left(y^{*} y^{*}\right) x^{*}\right)\right)^{*} \\
& =\left(\left(\left(y^{*} x^{*}\right) x^{*}\right)\left((0) x^{*}\right)\right)^{*} \\
& =\left(\left(\left(y^{*} x^{*}\right) x^{*}\right) 0\right)^{*} \\
& =\left(\left(\left(y^{*} x^{*}\right) x^{*}\right)\right)^{*} \\
& =\left(x(x y)^{*}\right)^{*}
\end{aligned}
$$

It follows from $\left(2^{*}\right)$ that $\mathcal{F}(x y)^{*} \geq \mathcal{F}\left(x(x y)^{*}\right)^{*}$, so from Theorem 3.18 that $\mathcal{F}$ is a fuzzy dot satisfactory filter of $X$.

Theorem 3.21. Let $\left\{\mathcal{F}_{i}\right\}$, where $i \in N$ be a family of fuzzy dot satisfactory filter of $X$, then so is $\cap_{i \in I} \mathcal{F}_{i}$.

Proof. Let for all $x, y \in X$, we get

$$
\begin{aligned}
\cap_{i \in I} \mathcal{F}_{i}(1) & =\min _{i \in I}\left\{\mathcal{F}_{i}(1)\right\} \\
& \geq \min _{i \in I}\left\{\mathcal{F}_{i}(x)\right\} \\
& =\cap_{i \in I} \mathcal{F}_{i}(x) \\
\cap_{i \in I} \mathcal{F}_{i}(y z)^{*} & =\min _{i \in I}\left\{\mathcal{F}_{i}(y z)^{*}\right\} \\
& \geq \min _{i \in I}\left\{\mathcal{F}_{i}\left(x\left(y(y z)^{*}\right)^{*}\right)^{*} \mathcal{F}_{i}(x)\right\} \\
\geq & \left(\min _{i \in I}\left\{\mathcal{F}_{i}\left(x\left(y(y z)^{*}\right)^{*}\right)^{*}\right\}\right)\left(\min _{i \in I}\left\{\mathcal{F}_{i}(x)\right\}\right. \\
& =\left(\cap_{i \in I} \mathcal{F}_{i}\left(x\left(y(y z)^{*}\right)^{*}\right)^{*}\right)\left(\cap_{i \in I} \mathcal{F}_{i}(x)\right)
\end{aligned}
$$

Hence $\cap_{i \in I} \mathcal{F}_{i}$ is a fuzzy dot satisfactory filter of $X$.

In the following example we can see if $\left\{\mathcal{F}_{i}\right\}$, where $i \in N$ is a family of fuzzy dot satisfactory filter of $X$, then $\cup_{i \in I} \mathcal{F}_{i}$ may not be a fuzzy dot satisfactory filter of $X$.

Example 3.23. Let $X=\{0, a, b, 1\}$ be a bounded $B C K$ algebra with $*$ defined as in Example 3.2. and let fuzzy dot satisfactory filter $v$ of $X$ which defined by $v(0)=0.3$, $v(a)=0.4$ and $v(b)=v(1)=0.5$. Defined the fuzzy subset $\lambda$ of $X$ by $\lambda(1)=\lambda(a)=1$ and $\lambda(0)=\lambda(b)=$ 
0.1 . Routine calculations give that $\lambda$ is a fuzzy dot filter of $X$. But $v \cup \lambda$ is not fuzzy dot filter, because:

$(v \cup \lambda)(10)^{*}=\max \left\{v(10)^{*}, \lambda(10)^{*}\right\}=0.3$

$<v \cup \lambda\left(\left(a\left(1(10)^{*}\right)^{*}\right)^{*}\right) v \cup \lambda(a)$

$=\max \left\{v\left(\left(a\left(1(10)^{*}\right)^{*}\right)^{*}\right), \lambda\left(\left(a\left(1(10)^{*}\right)^{*}\right)^{*}\right)\right\} \max \{v(a), \lambda(a)\}$

$=(\max \{0.1,0.5\})(\max \{1,0.4\} 0)=(0,5)(1)=0.5$

\section{CONCLUSiON}

The concept of fuzzy dot subalgebra of $B C K / B C I$ algebras was introduced by Jun and Hong paper [8]. In 2003 Jun [7] was introduced the concept of satisfactory $B C K$-filters. The concept of fuzzy satisfactory $B C K$-filters was introduced by Najati [13]. In this paper, we define the notion of fuzzy dot satisfactory $B C K$-filters and investigate some of its properties.

\section{REFERENCES}

[1] B. Ahmad, Dual ideals in BCK-algebra I, Math. Seminar Notes (presently, Kobe Jr. of Maths), 10 (1982), 243-250.

[2] B. Ahmad, Dual ideals in BCK-algebra II, Math. Seminar Notes, 10 (1982), 653-655.

[3] B. Ahmed, Characterizations of dual ideals in BCK-algebras, Math. Seminar Notes, 10 (1982), 647-652.

[4] E. Y. Deeba, A characterization of complete BCK-algebra, Math. Seminar Notes (presently, Kobe Jr. of Maths), 7 (1979), 343-349.

[5] Y. Imai and K. Ise'ki, On axiom systems of propositional calculi XIV, Proc. Japan Acad., 42 (1966), 26-29.

[6] K. Ise'ki, An algebra related with a propositional calculus, Proc. Japan Acad., 42 (1966), 351-366.

[7] Y. B. Jun, Satisfactory filters of BCK-algebras, Math. Japon., (9) (2003), 1-7.

[8] Y. B. Jun and S. M. Hong, Fuzzy subalgebras of BCK/BCIalgebras redfined, Math. Japon., 4\{2004), 769-775.

[9] Y. B. Jun, S. M. Hong and J. Meng, Fuzzy BCK-filters, Math. Japon., 47 (1) (1998), 45-49.

[10] Y. B. Jun, J. Meng and X. L. Xin, On fuzzy BCK-filters, Korean J. Comput. Appl. Math., 5 (1) (1998), 91-97.

[11] J. Meng, BCK-filters, Math. Japon., 44 (1996), 119-129.

[12] S. A. Najati, Fuzzy dot BCK-filters, IJSAR, 2(4) (2021), 1301-1303.

[13] S. A. Najati, Fuzzy satisfactory BCK-filters, IJSAR, 2(5) (2021), 1447-1450.

[14] J. Meng and Y. B. Jun, BCK-algebras, Kyungmoonsa Co. Korea, (1994).

[15] E. Roh and Y. B. Jun, On BCK-filters, Far East J. Math. Sci. II (1997), 181-188.

[16] O. G. Xi, Fuzzy BCK-algebras, Math. Japon., 36 (1991), 935942.

[17] L. A. Zadeh, Fuzzy sets, Inform. Control, 8 (1965), 338-353. 